\title{
STELLAR WINDS IN A-TYPE SUPERGIANTS
}

\author{
E.VERDUGO and A.TALAVERA* \\ IUE Observatory, European Space Agency \\ P.O.Box 50727, E-28080-Madrid, Spain
}

\section{Introduction}

A-type supergiants are just at the boundary between the early type supergiants, with strong stellar winds driven by radiation pressure and the cool supergiants in which the origin of mass loss is the dissipation of mechanical energy. The mechanisms involved in the mass loss processes in A supergiants as a whole, are still uncertain.

In a study of A-type supergiants observed with IUE, Talavera and Gomez de Castro (1987) divided them into two groups: Group I contained the less luminous A supergiants which show only weak signs of stellar winds. Group II are the most luminous A supergiants and they show strong evidences of wind and mass loss.

We present here a preliminary study of 33 stars in the visible and in the UV range, whose purpose is the modelling of the envelope-wind complex in A-type supergiants.

\section{2. $H \alpha$ and $H \beta$ profiles}

We have studied 29 stars of our sample in the visible range. The spectral intervals we have selected correspond to the following lines: CaII K line (3933 $\AA), \mathrm{MgII}(4481 \AA), \mathrm{H} \beta(4861 \AA), \mathrm{NaI}$ and $\mathrm{HeI}(5890 \AA)$ and $\mathrm{H} \alpha(6563 \AA)$.

The $\mathrm{H} \alpha$ profiles in A-type supergiants present very different shapes. The less luminous stars show symmetric absorption profiles but when luminosity increases the profile starts to be asymmetric becoming a P Cygni type III profile for the brightest stars (see Fig.1).

The less luminous stars do not show variability in the line profiles. However we have observed variations in some of the stars with asymmetricemission profiles. In five "peculiar" stars the observed profile shows very strong variations, becoming in two stars a pure emission profile.

In most of the stars the $\mathrm{H} \beta$ observed profile does not show evidence of mass loss. However in the five "peculiar" stars with very strong variations in $\mathrm{H} \alpha$, the $\mathrm{H} \beta$ profile is asymmetric and shows emission (see Fig.1).

- Affiliated to the Astrophysics Division, Space Science Department 

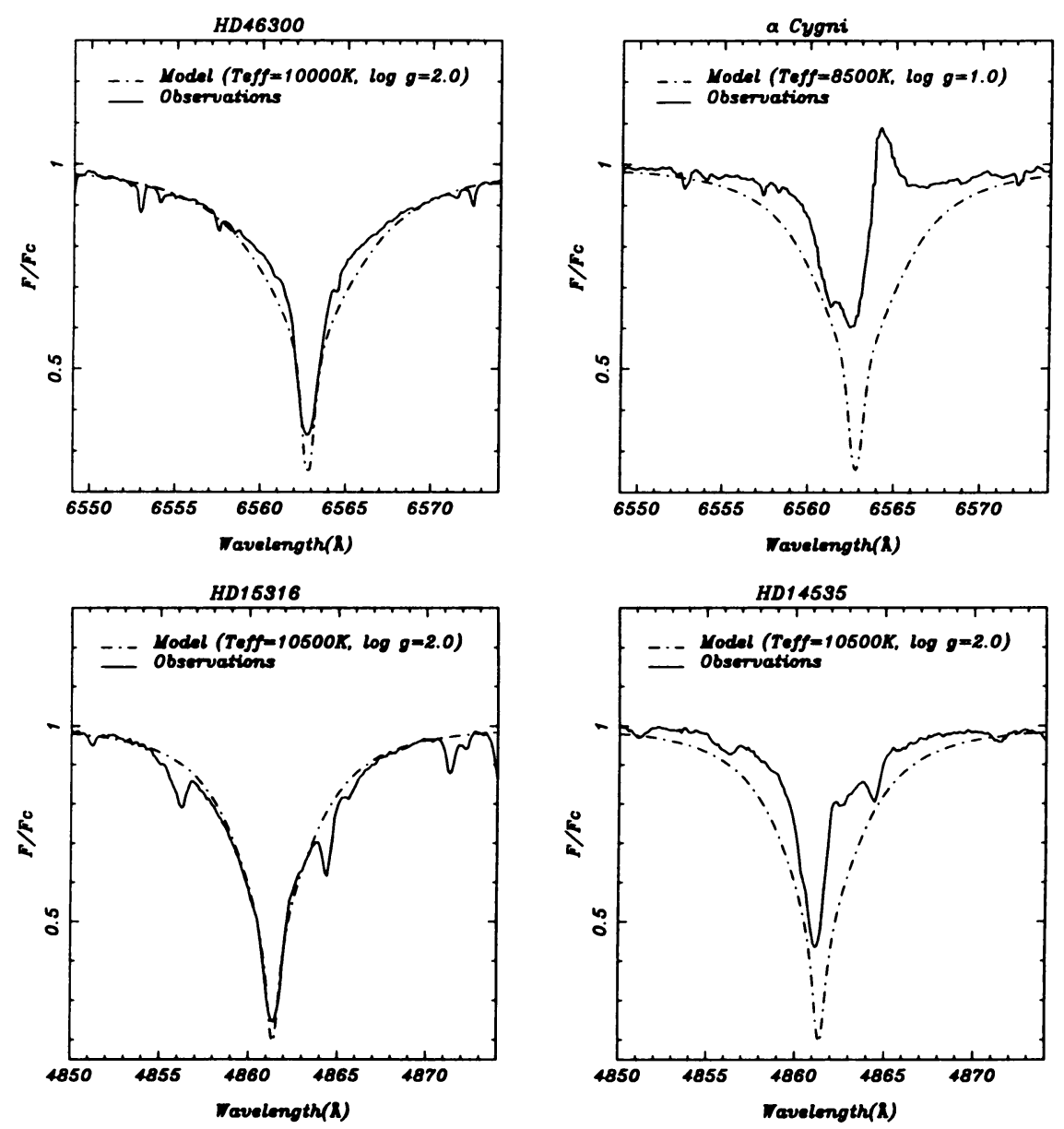

Fig. 1. Effects on the $\mathrm{H} \alpha$ (above) and $\mathrm{H} \beta$ (below) profiles of the wind

\section{Model Atmospheres}

We have fitted the energy distribution (visible plus uv) using the Kurucz model atmospheres as a preliminary solution. These models assume LTE and radiative equilibrium, and include line blanketing in the opacity.

For stars with mass loss the Kurucz models are inadequate for a detailed explanation of spectral features formed in the wind (see Fig.1).

In the second part of our project we shall try the modelling of these winds taking into account effects of velocity fields in expanding atmospheres.

\section{References}

Kurucz, R.L.: 1979a, Ap. J. Suppl. 40, 1

Talavera, A. and Gomez de Castro, A.I.: 1987, Astron. Astrophys. 181, 300 